\title{
ELIMINACIÓN DE COLORANTES CATIÓNICOS USANDO OZONO, ZEOLITA NATURAL Y OZONO/ZEOLITA
}

\section{CATIONIC DYES REMOVAL USING OZONE, NATURAL ZEOLITE, AND OZONE/ZEOLITE}

\author{
Héctor Valdés ${ }^{1} \quad$ Rolando F. Tardón ${ }^{2} \quad$ Claudio A. Zaror ${ }^{2}$ \\ Recibido 10 de junio de 2008, aceptado 11 de septiembre de 2009 \\ Received: June 10, 2008 Accepted: September 11, 2009
}

\begin{abstract}
RESUMEN
En este trabajo se comparan resultados experimentales de remoción azul de metileno (MB) utilizando tratamientos basados en la oxidación con ozono $\left(\mathrm{O}_{3}\right)$, la adsorción con zeolita natural $(\mathrm{ZN})$, y tratamiento simultáneo de adsorción y oxidación con ozono en presencia de zeolita natural $\left(\mathrm{O}_{3} / \mathrm{ZN}\right)$. Se evalúa, a escala de laboratorio, el efecto del $\mathrm{pH}(2-8)$ y la presencia de sustancias atrapadoras radicales libres (iones acetatos) en la velocidad de remoción y en la eficiencia de los procesos. Los experimentos se realizaron en un reactor diferencial compuesto por un estanque de $1 \mathrm{dm}^{3}$ y una columna de $19 \mathrm{~cm}^{3} \mathrm{de}$ capacidad. El ozono fue generado a razón de $5 \mathrm{~g} \mathrm{O}_{3} / \mathrm{h}$. Los resultados mostraron que el sistema simultáneo de oxidación/ adsorción $\mathrm{O}_{3} / \mathrm{ZN}$ incrementa la velocidad de remoción del $\mathrm{MB}$ con respecto a los procesos separados de ozonización y adsorción con zeolita. En presencia de sustancias atrapadoras de radicales, se observó un $70 \%$ de disminución en la velocidad de remoción de $\mathrm{MB}$ cuando se empleó el tratamiento con $\mathrm{O}_{3}$ y sólo un $25 \%$ cuando se utiliza el tratamiento combinado $\mathrm{O}_{3} / \mathrm{ZN}$. Los resultados sugieren que la reacción de oxidación del MB en el sistema tiene lugar fundamentalmente sobre la superficie de la zeolita.
\end{abstract}

Palabras clave: Adsorción, azul de metileno, colorantes catiónicos, ozono, ozonización catalítica, zeolita natural.

\section{ABSTRACT}

This paper compares experimental results on methylene blue $(M B)$ removal systems based on ozone oxidation, zeolite adsorption, and simultaneous adsorption-oxidation using ozone in the presence of natural zeolite. The effect of pH (2-8), and the presence of radical scavengers (sodium acetate) on process rates and removal efficiencies are assessed at laboratory scale. The experimental system consisted of a $1 \mathrm{~L}$ differential circular flow reactor and an ozone generator rated at $5 \mathrm{~g}$ $\mathrm{O}_{3} / \mathrm{h}$. Results show that ozone oxidation combined with zeolite adsorption increases the overall MB oxidation rate with respect to ozonation process and zeolite adsorption. In presence of free radical scavenger, only a $25 \%$ of reduction on $M B$ removal rate are observed in the simultaneous treatment, as compared with $70 \%$ when ozonation treatment is used, suggesting that MB oxidation reactions take mainly place on the zeolite surface.

Keywords: Adsorption, cationic dyes, catalytic ozonation, methylene blue, ozone, natural zeolite.

\section{INTRODUCCIÓN}

La nocividad y persistencia en el tiempo de algunos colorantes orgánicos catiónicos imposibilita su tratamiento mediante métodos convencionales. El uso de los Procesos de Oxidación Avanzada (AOPs) surge como una opción atractiva de tratamiento, debido a la alta capacidad de oxidación. Los AOPs se basan fundamentalmente en la formación de radicales libres caracterizados por su alta reactividad y no selectividad. Sin embargo, la presencia de agentes inhibidores que se encuentran normalmente presentes en las aguas, tales como carbonatos y bicarbonatos, disminuye la eficiencia de degradación de estos procesos.

De manera de aumentar la eficiencia de los AOPs se ha planteado un nuevo proceso heterogéneo que combina la acción simultánea del carbón activado y el ozono en un mismo proceso de tratamiento, incrementando la generación de radicales libres [1-3]. El alto costo adquisitivo del carbón activado muestra ser una limitante

\footnotetext{
1 Facultad de Ingeniería. Universidad Católica de la Santísima Concepción. Alonso de Ribera 2850. Concepción, Chile. E-mail: hvaldes@ ucsc.cl
}

2 Departamento de Ingeniería Química. Universidad de Concepción. Edmundo Larenas s/n. Concepción, Chile. E-mail: czaror@udec.cl 
en la implementación de esta tecnología a gran escala. Por otro lado, el uso de materiales naturales, como zeolitas ha manifestado ser una alternativa atractiva al carbón activado como soporte más económico en los procesos oxidativos y adsortivos [4-5].

El estudio que se presenta tuvo como objetivo comparar la eliminación de un colorante catiónico utilizando tratamientos basados en el uso de: ozono $\left(\mathrm{O}_{3}\right)$, zeolita natural $(\mathrm{ZN})$, tratamiento combinado ozono/zeolita natural $\left(\mathrm{O}_{3} / \mathrm{ZN}\right)$. Como contaminante modelo se utilizó azul de metileno (MB), compuesto aromático heterocíclico catiónico, que presenta una difícil biodegradación por métodos biológicos convencionales, debido a su propiedad bactericida. Se analiza el efecto del $\mathrm{pH}$ y de la presencia de sustancias atrapadoras de radicales libres en la velocidad y en la eficiencia de remoción.

\section{METODOLOGÍA}

En el estudio se empleó zeolita natural suministrada por la compañía minera "Minera Formas", con un tamaño de partículas promedio en el rango 0.60-0.85 mm. Esta zeolita está compuesta de clinoptilolita (53\%), mordenita (40\%), y cuarzo (7\%). En la Tabla 1 se muestran las características físico-químicas de la zeolita utilizada.

Tabla 1. Características físico-químicas de la zeolita natural utilizada.

\begin{tabular}{|l|c|}
\hline $\mathrm{S}_{\mathrm{N} 2}\left(\mathrm{~m}^{2} / \mathrm{g}\right)^{\mathrm{a}}$ & 205 \\
\hline $\mathrm{pH}_{\mathrm{PZ}}{ }^{\mathrm{b}}$ & 7.9 \\
\hline Sitios ácidos $(\mu \mathrm{eq}-\mathrm{g} / \mathrm{g})^{\mathrm{c}}$ & 220 \\
\hline Sitios básicos $(\mu \mathrm{eq}-\mathrm{g} / \mathrm{g})^{\mathrm{c}}$ & 960 \\
\hline $\mathrm{SiO}_{2}(\%)^{\mathrm{d}}$ & 75.0 \\
\hline $\mathrm{Al}_{2} \mathrm{O}_{3}(\%)^{\mathrm{d}}$ & 14.86 \\
\hline $\mathrm{CaO}(\%)^{\mathrm{d}}$ & 4.40 \\
\hline $\mathrm{Fe}_{2} \mathrm{O}_{3}(\%)^{\mathrm{d}}$ & 2.02 \\
\hline $\mathrm{MgO}^{\mathrm{d}}(\%)^{\mathrm{d}}$ & 1.01 \\
\hline $\mathrm{K}_{2} \mathrm{O}(\%)^{\mathrm{d}}$ & 0.72 \\
\hline $\mathrm{TiO}_{2}(\%)^{\mathrm{d}}$ & 0.36 \\
\hline $\mathrm{Na}_{2} \mathrm{O}(\%)^{\mathrm{d}}$ & 1.51 \\
\hline $\mathrm{PI}^{\mathrm{d}}(\%)^{\mathrm{e}}$ & 11.43 \\
\hline
\end{tabular}

a Area superficial determinada por adsorción de $\mathrm{N}_{2}$ a $77 \mathrm{~K}$.

b Determinado por valoración ácido-base con $\mathrm{NaOH}(0.1 \mathrm{M})$ o $\mathrm{HCl}$ $(0.1 \mathrm{M})$, en presencia de $\mathrm{NaCl}$ como electrólito inerte $\left(10^{-1} \mathrm{M}, 10^{-2}\right.$ $\left.\mathrm{M}, 10^{-3} \mathrm{M}\right)$.

c Concentración de sitios activos determinados por valoración ácidobase con $\mathrm{NaOH}(0.1 \mathrm{M})$ o $\mathrm{HCl}(0.1 \mathrm{M})$.

d Determinados por fluorescencia de rayos-X.

e Pérdida por ignición.
Los experimentos fueron realizados a $20^{\circ} \mathrm{C}$, en un sistema de reacción compuesto por una columna de $19 \mathrm{~cm}^{3}$, cargada zeolita, y un estanque de $1 \mathrm{dm}^{3}$ de capacidad. El reactor fue operado en régimen discontinuo. El ozono fue producido in situ a partir de oxígeno puro y seco, utilizando un generador de ozono $\left(5 \mathrm{~g} \mathrm{O}_{3} / \mathrm{h}\right)$. El valor del $\mathrm{pH}$ de la solución fue ajustado a 2, 4, 6 y 8 utilizando ácido clorhídrico $(1.0 \mathrm{M})$ o hidróxido de sodio $(1.0 \mathrm{M})$, con una solución tampón de fosfato de sodio $\left(5 \times 10^{-3} \mathrm{M}\right)$.

Una vez alcanzado el valor de la saturación de ozono en solución (aproximadamente $30 \mathrm{~min}$ ), se cerró la válvula de alimentación de ozono gaseoso, al mismo tiempo se procedió a la inyección de una solución concentrada de MB de $10 \mathrm{~g} / \mathrm{dm}^{3}$ dentro del reactor y a la recirculación de líquido a través del lecho fijo cargado con la zeolita. Para soportar la zeolita en el reactor se utilizó lana de vidrio. La concentración de ozono y MB en solución fueron monitoreadas por espectrofotometría UV, empleando una celda de flujo, midiendo el cambio en la absorción a $258 \mathrm{~nm}$ y $658 \mathrm{~nm}$, respectivamente.

\section{RESULTADOS Y DISCUSIÓN}

\section{Comparación del tratamiento de remoción de $\mathrm{MB}$ basado en el uso de: ozono $\left(\mathrm{O}_{3}\right)$, zeolita natural $(\mathrm{ZN})$, ozono/zeolita $\left(\mathrm{O}_{3} / \mathrm{ZN}\right)$}

La Figura 1 muestra la disminución adimensional del MB en función del tiempo a $20^{\circ} \mathrm{C}, \mathrm{pH} 2$, a una concentración inicial de $\mathrm{MB}$ de $30 \mathrm{mg} / \mathrm{dm}^{3}$, cuando se utiliza tratamiento con $\mathrm{O}_{3}$ (concentración inicial de ozono de $6 \mathrm{mg} / \mathrm{dm}^{3}$ ), zeolita natural $\left(15 \mathrm{~g} / \mathrm{dm}^{3}\right)$ y el tratamiento $\mathrm{O}_{3} / \mathrm{ZN}$ $\left(15 \mathrm{~g} / \mathrm{dm}^{3}\right.$ zeolita natural, concentración inicial de ozono de $\left.6 \mathrm{mg} / \mathrm{dm}^{3}\right)$.

Según se aprecia en la Figura 1, es posible observar que el tratamiento combinado aumenta sustancialmente la eliminación de $\mathrm{MB}$, teniendo un efecto sinérgico en comparación a los procesos individuales con $\mathrm{O}_{3}$ y ZN. Los datos experimentales se ajustaron a un modelo cinético aparente de pseudoprimer orden con respecto a la concentración de MB. El valor de la constante de velocidad observada de pseudoprimer orden de eliminación del MB varió desde $0.3 \times 10^{-3} \mathrm{~s}^{-1}$ para el tratamiento con $\mathrm{O}_{3}$, a $1.2 \times 10^{-3} \mathrm{~s}^{-1}$ cuando se realiza con $\mathrm{ZN}$, alcanzando un valor máximo de $1.8 \times 10^{-3} \mathrm{~s}^{-1}$ cuando se utiliza el tratamiento combinado $\mathrm{O}_{3} / \mathrm{ZN}$. El aumento en la velocidad de remoción en el tratamiento combinado no sólo se debería a la contribución individual de cada mecanismo, sino también a que la zeolita original contribuye a la descomposición del ozono en fase acuosa. 
Los sitios óxidos metálicos superficiales actuarían como iniciadores y promotores de la reacción en cadena de radicales libres, reacción que continúa en el seno de la solución y acelera la descomposición del ozono en oxidantes secundarios [6].

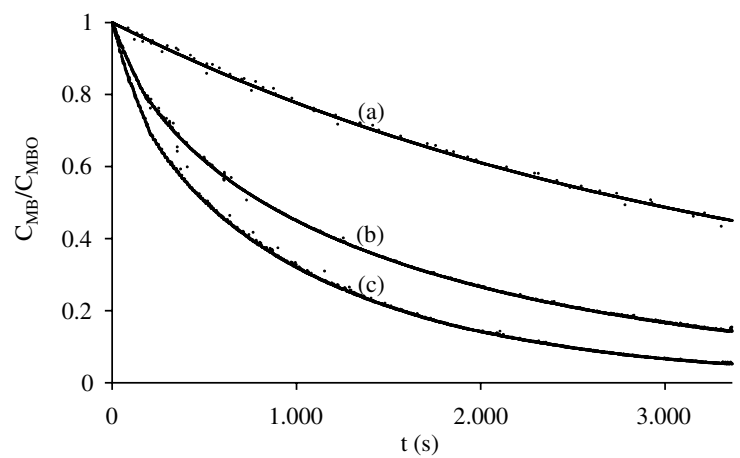

Figura 1. Comparación en la degradación de MB por tres tipos de tratamientos: (a) $\mathrm{O}_{3}$, (b) $\mathrm{ZN}$, (c) $\mathrm{O}_{3} / \mathrm{ZN}$. Condiciones de operación: $\mathrm{pH} 2$, $30 \mathrm{mg} / \mathrm{dm}^{3}$ de concentración inicial de MB, $6 \mathrm{mg} / \mathrm{dm}^{3}$ de concentración inicial de ozono, $15 \mathrm{~g} / \mathrm{dm}^{3}$ de zeolita natural.

\section{Efecto del pH}

La Figura 2 muestra el efecto del pH (2-8) en la remoción de $\mathrm{MB}$, para una concentración inicial de $\mathrm{MB}$ de $30 \mathrm{mg} / \mathrm{dm}^{3}$, concentración inicial de ozono disuelto de $6 \mathrm{mg} / \mathrm{dm}^{3}$, en el tratamiento con $\mathrm{O}_{3}$ y en el sistema combinado $\mathrm{O}_{3} / \mathrm{ZN}$ a $20^{\circ} \mathrm{C}$.

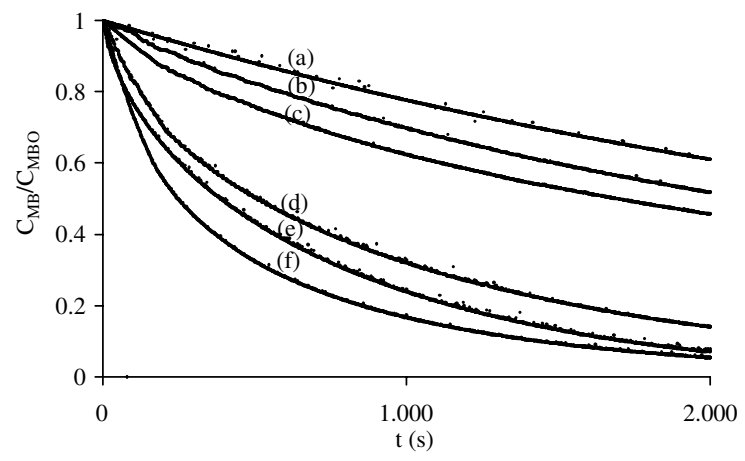

Figura 2. Efecto del pH en la velocidad de remoción de $\mathrm{MB}$ en los tratamientos con $\mathrm{O}_{3}$ y $\mathrm{O}_{3} / \mathrm{ZN}$. $\mathrm{O}_{3}$ : (a) pH 2, (b) pH 6, (c) pH 8. O 3 /zeolite: (d) $\mathrm{pH} 2$, (e) $\mathrm{pH} 6$, (f) $\mathrm{pH} 8$. Condiciones de operación: $20^{\circ} \mathrm{C}, 30 \mathrm{mg} / \mathrm{dm}^{3}$ de concentración inicial de $\mathrm{MB}, 6 \mathrm{mg} / \mathrm{dm}^{3}$ de concentración inicial de ozono disuelto y $15 \mathrm{~g} / \mathrm{dm}^{3}$ zeolita natural.

Según se aprecia en la Figura 2, la disminución en la concentración adimensional de MB se incrementó con el aumento del $\mathrm{pH}$. La presencia de zeolita natural incrementó la velocidad de remoción del MB para cada nivel de $\mathrm{pH}$ estudiado. Los datos experimentales se ajustaron a un modelo cinético aparente de pseudoprimer orden con respecto a la concentración de $\mathrm{MB}\left(\mathrm{R}^{2}>0.99\right)$. Los valores experimentales observados de la constante de velocidad de pseudoprimer orden de eliminación del MB aumentaron desde $0.3 \times 10^{-3} \mathrm{~s}^{-1}$ a $1.8 \times 10^{-3} \mathrm{~s}^{-1} ; 0.5 \times 10^{-3} \mathrm{~s}^{-1} \mathrm{a}$ $2.6 \times 10^{-3} \mathrm{~s}^{-1} ; 0.7 \times 10^{-3} \mathrm{~s}^{-1}$ a $3.1 \times 10^{-3} \mathrm{~s}^{-1}$ para $\mathrm{pH} 2$, 6 y 8 , respectivamente, cuando el tratamiento se realiza sólo con $\mathrm{O}_{3}$ a cuando se utiliza el tratamiento combinado $\mathrm{O}_{3} / \mathrm{ZN}$.

Los resultados obtenidos en el transcurso de la experimentación indican que la capacidad de adsorción del MB no es afectada por el cambio de $\mathrm{pH}$. Sin embargo, en el tratamiento combinado $\mathrm{O}_{3} / \mathrm{ZN}$ la velocidad de remoción de MB se incrementa con el aumento del pH más allá de los valores obtenidos con el tratamiento con $\mathrm{O}_{3}$.

El incremento en la velocidad de remoción del MB se puede relacionar con la interacción del ozono y la superficie de la zeolita natural. Según se indica en una publicación reciente, el incremento en la velocidad de descomposición del ozono en presencia de zeolita natural se atribuye a una combinación de descomposición catalizada por la superficie de la zeolita y a la participación en reacciones químicas con los sitios ácidos de Lewis presentes en la superficie de la zeolita natural [7]. Para cada nivel de pH el incremento en la velocidad de descomposición del ozono está vinculado con la interacción del ozono y los sitios superficiales activos de la zeolita. El ozono muestra diferente grado de reactividad hacia los sitios óxidos metálicos de la superficie de la zeolita con valores de carga superficiales que varían en función al $\mathrm{pH}$ de la solución [7].

Tabla 2. Efecto del pH en la velocidad de remoción de MB.

\begin{tabular}{|l|c|c|c|}
\hline \multirow{2}{*}{ Procesos } & \multicolumn{3}{|c|}{$\begin{array}{c}\text { Constante aparente de velocidad de } \\
\text { pseudoprimer orden } \times 10^{3}\left(\mathrm{~s}^{-1}\right)\end{array}$} \\
\cline { 2 - 4 } & $\mathrm{pH} 2$ & $\mathrm{pH} 6$ & $\mathrm{pH} 8$ \\
\hline $\mathrm{O}_{3}$ & 0.3 & 0.5 & 0.7 \\
\hline $\mathrm{ZN}$ & 1.2 & 1.2 & 1.2 \\
\hline $\mathrm{O}_{3} / \mathrm{ZN}$ & 1.8 & 2.6 & 3.1 \\
\hline
\end{tabular}

Para condiciones de $\mathrm{pH}>\mathrm{pH}_{\mathrm{PZC}}$, la superficie de la zeolita adquiere carga negativa. Bajo esas condiciones de $\mathrm{pH}$, los grupos ácidos disociados presentes en la superficie de la zeolita incrementarían la velocidad de reacción con el ozono, catalizando la formación de radicales hidroxilos y con ello el aumento en la velocidad de remoción de MB. 
Efecto de la presencia de sustancias atrapadoras de radicales libres

Se realizaron experimentos en presencia y ausencia de iones acetatos $\left(\mathrm{Ac}^{-}\right), \mathrm{pH} 8,20^{\circ} \mathrm{C}$, concentración inicial de MB de $30 \mathrm{mg} / \mathrm{L}$, y saturación inicial de ozono de $6 \mathrm{mg} / \mathrm{L}$. Los iones acetatos son conocidos como atrapadores de radicales libres, reaccionan rápidamente con los radicales hidroxilos $\left(\mathrm{k}_{\mathrm{OH} \cdot / \mathrm{Ac}}{ }^{-}=8 \times 10^{7} \mathrm{M}^{-1} \cdot \mathrm{s}^{-1}\right)$ y de forma lenta con el ozono $\left(\mathrm{k}_{\mathrm{O} 3 \cdot / \mathrm{Ac}}{ }^{-1} 10^{-4} \mathrm{M}^{-1} \cdot \mathrm{s}^{-1}\right)[8]$.

A partir de los resultados mostrados en la Figura 3 se ajustaron los datos experimentales a un modelo cinético aparente de pseudoprimer orden con respecto a la concentración de $\mathrm{MB}\left(\mathrm{R}^{2}>0.99\right)$. Se obtuvo que a $\mathrm{pH} 8$, la constante de velocidad de remoción de MB descendió desde $0.7 \times 10^{-3} \mathrm{~s}^{-1}$ a $0.2 \times 10^{-3} \mathrm{~s}^{-1}$ en presencia de iones acetatos cuando el tratamiento se realiza con $\mathrm{O}_{3}$.

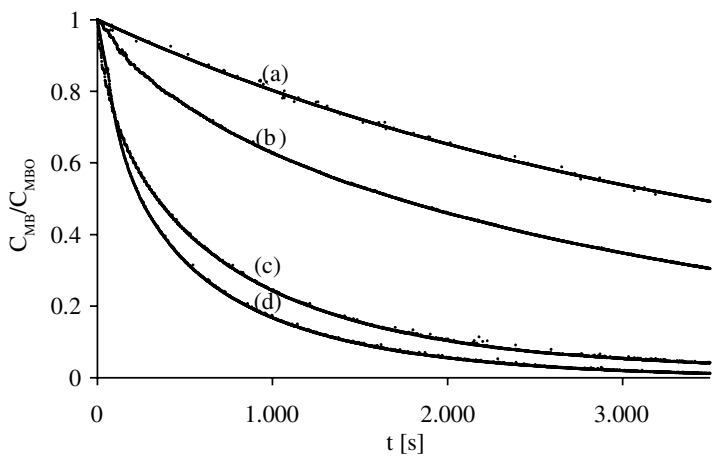

Figura 3. Efecto de la presencia y ausencia de iones acetatos en la velocidad de remoción de $\mathrm{MB}$ en los tratamientos con $\mathrm{O}_{3}$ y $\mathrm{O}_{3} / \mathrm{ZN}$. $\mathrm{O}_{3}$ : (a) $0.1 \mathrm{M} \mathrm{Ac}^{-}$, (b) $0 \mathrm{M} \mathrm{Ac}^{-}$. $\mathrm{O}_{3} / \mathrm{ZN}$ : (c) $0.1 \mathrm{M}$ $\mathrm{Ac}^{-}$, (d) $0 \mathrm{M} \mathrm{Ac}^{-}$. Condiciones de operación: $20^{\circ} \mathrm{C}, \mathrm{pH} 8,30 \mathrm{mg} / \mathrm{dm}^{3}$ de concentración inicial de $\mathrm{MB}, 15 \mathrm{~g} / \mathrm{dm}^{3}$ zeolita natural.

$\mathrm{Al}$ aplicar el tratamiento combinado $\mathrm{O}_{3} / \mathrm{ZN}$ la constante de velocidad de remoción de MB descendió desde $3.1 \times 10^{-3} \mathrm{~s}^{-1}$ a $2.3 \times 10^{-3} \mathrm{~s}^{-1}$ en presencia de iones acetatos. Lo anterior indica un $70 \%$ de disminución en la velocidad de remoción de MB debido a la presencia de iones acetatos cuando se emplea tratamiento con $\mathrm{O}_{3}$, y sólo un $25 \%$ cuando se utiliza el sistema combinado $\mathrm{O}_{3} / \mathrm{ZN}$.
Tabla 3. Efecto de la presencia de iones acetato en la velocidad de remoción de MB.

\begin{tabular}{|l|c|c|}
\hline \multirow{2}{*}{ Procesos } & \multicolumn{2}{|c|}{$\begin{array}{c}\text { Constante aparente de pseudoprimer } \\
\text { orden } \times 10^{3}\left(\mathrm{~s}^{-1}\right)\end{array}$} \\
\cline { 2 - 3 } & $\begin{array}{c}\text { Sin atrapador de } \\
\text { radicales }\end{array}$ & $\begin{array}{c}\text { Con atrapador de } \\
\text { radicales }\end{array}$ \\
\hline $\mathrm{O}_{3}$ & 0.7 & 0.2 \\
\hline $\mathrm{O}_{3} / \mathrm{ZN}$ & 3.1 & 2.3 \\
\hline
\end{tabular}

Estos resultados sugieren que la reacción de oxidación del MB tiene lugar fundamentalmente sobre la superficie de la zeolita. El mecanismo de reacción pudiera estar determinado por reacciones superficiales, con los grupos superficiales hidroxilos unidos a los sitios óxidos metálicos actuando como iniciadores y/o promotores de las reacciones en cadena de los radicales libres, acelerando la descomposición del ozono en el seno de la solución.

\section{CONCLUSIONES}

El uso de $\mathrm{O}_{3}$, ZN y $\mathrm{O}_{3} / \mathrm{ZN}$ resulta efectivo para el tratamiento de aguas contaminadas con colorantes orgánicos catiónicos. El tratamiento simultáneo $\mathrm{O}_{3} / \mathrm{ZN}$ incrementa la velocidad de remoción, comparado con los tratamientos de oxidación simple con ozono y de adsorción con zeolita natural. El valor de la constante de velocidad observada de pseudoprimer orden de eliminación del MB varió desde $0,3 \times 10^{-3} \mathrm{~s}^{-1}$ para el tratamiento con $\mathrm{O}_{3}$, a $1.2 \times 10^{-3} \mathrm{~s}^{-1}$ cuando se realiza con $\mathrm{ZN}$, alcanzando un valor máximo de $1.8 \times 10^{-3} \mathrm{~s}^{-1}$ cuando se utiliza el tratamiento combinado $\mathrm{O}_{3} / \mathrm{ZN}$.

Para cada nivel de $\mathrm{pH}$ estudiado, la zeolita natural incrementa la velocidad de remoción del MB. Este incremento pudiera estar relacionado con la fracción de grupos hidroxilos unidos a los óxidos superficiales presentes en la zeolita que exhiben cambios en los valores de la carga superficial en función del $\mathrm{pH}$ de la solución. Para condiciones de $\mathrm{pH}>\mathrm{pH}_{\mathrm{PZC}}$, los grupos superficiales ácidos disociados presentes en la zeolita pudieran incrementar la velocidad de reacción con el ozono, aumentando la velocidad de remoción del MB.

Los resultados demuestran la importancia de las reacciones por radicales libres en el tratamiento combinado $\mathrm{O}_{3} / \mathrm{ZN}$. En el tratamiento simultáneo, el efecto de las sustancias atrapadoras de radicales libres es reducido, sugiriendo que la superficie de la zeolita juega un papel fundamental en el mecanismo de la reacción, y que los radicales libres pueden estar presentes tanto en la interfase sólido-líquido como en el seno de la solución. 
La zeolita natural chilena es un material de bajo costo que se puede utilizar en conjunto con ozono para la eliminación de colorantes catiónicos a través de un proceso de ozonización catalítico heterogéneo.

\section{AGRADECIMIENTOS}

Los autores agradecen a FONDECYT (Proyectos $\mathrm{N}^{\circ}$ 1060304 y 1060892) por el apoyo brindado en el desarrollo de la investigación.

\section{REFERENCIAS}

[1] H. Valdés and C.A. Zaror. "Advanced treatment of benzothiazole contaminated waters: comparation of $\mathrm{O}_{3}, \mathrm{AC}$, and $\mathrm{O}_{3} / \mathrm{AC}$ processes". Water Sci.Technol. Vol. 52, pp. 281-288. 2005.

[2] H. Valdés and C.A. Zaror. "Heterogeneous and Homogeneous Catalytic Ozonation of Benzothiazole Promoted by Activated Carbon: Kinetic Approach". Chemosphere. Vol. 65, pp. 1131-1136. 2006.

[3] H. Valdés and C.A. Zaror. "Ozonation of Benzothiazole Saturated-Activated carbons: Influence of Carbon Chemical Surface Properties".
J. Hazard. Mater. Vol. 137, pp. 1042-1048. 2006.

[4] H. Fujita. "Adsorbed phase ozonation of waterdissolved organic pollutants using high-silica zeolitas". Adsorption. Vol. 11, pp. 835-839. 2005.

[5] H.M. Ötker and I. Akmehmet-Balcıŏlu. "Adsorption and degradation of enrofloxacin, a veterinary antibiotic on natural zeolite". J. Hazard. Mater. Vol. 122, pp. 251-258. 2005.

[6] H. Valdés, F.A. Murillo, J.A. Manoli and C.A. Zaror. "Heterogeneous catalytic ozonation of benzothiazole aqueous solution promoted by volcanic sand". J. Hazard. Mater. Vol. 153, pp. 1036-1042. 2008.

[7] H. Valdés, V.J. Farfán, J.A. Manoli and C.A. Zaror. "Catalytic ozone aqueous decomposition promoted by natural zeolite and volcanic sand". J. Hazard. Mater. Vol. 165, pp. 915-922. 2009.

[8] U. Jans and J. Hoigné. "Activated carbon and carbon black catalyzed transformation of aqueous ozone into OH-radicals". Ozone: Sci. Eng. Vol. 20, pp. 67-89. 1998. 